The Centre for Global Vaccine Research, Institute of Infection, Veterinary and Ecological Sciences, University of Liverpool, L69 7BE, Liverpool, UK

2 NIHR Health Protection Research Unit in Gastrointestinal Infections, University of Liverpool, UK

Correspondence to: D Hungerford d.hungerford@liverpool.ac.uk Cite this as: BMJ2021;374:n2034 http://dx.doi.org/10.1136/bmj.n2034 Published: 20 August 2021

\section{Real world effectiveness of covid-19 vaccines}

\author{
Rigorous studies of these vaccines in action are an urgent priority globally \\ Daniel Hungerford, ${ }^{1,2}$ Nigel A Cunliffe, 2
}

Post-introduction vaccine studies provide practitioners and policy makers with the kind of evidence that clinical trials cannot-including real world vaccine effectiveness against multiple clinical outcomes. Two linked studies ${ }^{12}$ that together examined three covid-19 vaccines, add to mounting evidence that efficacy reported in clinical trials translates well to the real world. $3^{-7}$ The studied vaccines-CoronaVac (Sinovac Biotech), BNT162b2 (Pfizer-BioNTech), and mRNA-1273

(Moderna)-performed well in preventing severe covid-19 and deaths in adults after two doses; a single dose was significantly less effective, and all three vaccines were effective (although demonstrably less so) against milder disease.

Both studies used test negative case-control designs to estimate effectiveness-comparing vaccination history among people with symptoms who tested either positive (cases) or negative (controls) for SARS-CoV-2. Ranzani and colleagues (doi:10.1136/bmj.n2015) evaluated CoronaVac in adults aged 70 or older in Brazil during a gamma variant epidemic and report an effectiveness after two doses of $42 \%$ against symptomatic covid- 19 of any severity. ${ }^{1}$ Chung and colleagues (doi:10.1136/bmj.n1943) evaluated two mRNA vaccines (BNT162b2 and mRNA-1273) in adults in Canada, reporting point estimates of greater than $80 \%$ effectiveness after two doses against symptomatic covid-19 caused by several variants-including alpha, beta, and gamma-but lower effectiveness (43-61\%) after a single dose. ${ }^{2}$ Additional studies of delta and other variants will be needed as SARS-CoV-2 continues to evolve. ${ }^{8}$

Vaccine performance is highly context dependent, influenced by population risk of infection and disease, so assessment is required among multiple different subgroups. Importantly, the Brazilian study added to age restricted clinical trials by including only adults aged 70 or older, reporting $80 \%$ effectiveness against hospital admissions after two doses in 70-74 year olds but declining effectiveness with increasing age. Both new studies provide evidence that delaying the second dose beyond manufacturers' recommended schedules might be beneficial for some subgroups. For example, populations at low risk of complicated covid-19 benefit because protection against severe disease increases with time after the first dose.

The need to evaluate different covid-19 vaccines against multiple endpoints and variants in a range of subgroups means that effectiveness studies will be a staple of public health and academic workload for the foreseeable future. We need to ensure that methodological approaches such as test negative case-control designs can be delivered through common protocols using routine, quality controlled, health and surveillance data systems. Harmonisation will minimise the proliferation of expensive bespoke studies with individualised designs requiring different approaches to data collection and ethical approval, thereby protecting scarce resources for other public health priorities.

Additionally, as countries reach high levels of vaccine coverage and case rates fall, case-control designs will become more challenging. ${ }^{9}$ Alternative approaches such as cohort studies offer opportunities to explore indirect effects of vaccination, including herd protection, and the effects of covid-19 vaccines on non-specific outcomes, such as absenteeism from work or school and symptoms such as gastrointestinal illness. These designs have limitations, however, including the potential for confounding as a result of important differences between unvaccinated and vaccinated groups-differences in health status, access to healthcare, and health seeking behaviour, for example. ${ }^{10} 11$ Ultimately, vaccine effectiveness studies are only as good as the data available, and measures to capture high quality, timely data must be prioritised, particularly in low and middle income countries where disease burdens are high and systems for routine data collection can be suboptimal.

Since covid-19 vaccines were authorised for use on accelerated regulatory pathways with comparatively little supportive clinical data, robust and timely real world safety data are critical to inform risk, minimise harm, and maintain public confidence. This was recently highlighted in the context of the ChAdOx1 nCoV-19 (Oxford-AstraZeneca) vaccine by the paucity of accurate data on background rates of thromboembolic events to inform the response to an emerging safety signal. ${ }^{12} 13$

In some countries, vaccines are offered to most of the population, including both high risk individuals and those targeted primarily because of their role in transmission. This latter group includes children in Canada, the USA, Indonesia, Chile, and Malta. ${ }^{14-16}$ Data on the short term safety of covid-19 vaccines in young children are emerging from clinical studies. ${ }^{17}$ Nevertheless, considering that severe covid-19 is less common in children than in adults, it is imperative that rigorous long term safety and effectiveness studies are conducted in children to quantify risk-benefit balance and inform vaccine policy and choices.

The success of any vaccine programme depends on the quality of real world evaluations. Decisions on which vaccines to purchase and which groups to target must be based on the highest quality analyses of vaccines in action, fully contextualised according 
to place and population and accounting for all relevant biases. We must also facilitate timely access to expertise and data in countries with greatest disease burden, to ensure optimal performance of covid-19 vaccines and equity globally.

The BMJ has judged that there are no disqualifying financial ties to commercial companies. The authors declare the following other interests: $\mathrm{DH}$ and NC have contributed to commercially funded studies on rotavirus vaccines.

Further details of The BMJ policy on financial interests is here: https:/www.bmj.com/sites/default/files/attachments/resources/2016/03/16-current-bmj-education-coi-form.pdf."

Provenance and peer review: Commissioned; not peer reviewed.

1 Ranzani OT, Hitchings MDT, Dorion M, etal. Effectiveness of the CoronaVac vaccine in older adults during a gamma variant associated epidemic of covid-19 in Brazil: test negative case-control study. BMJ2021;374:n2015.

2 Chung H, He S, Nasreen S, etalCanadian Immunization Research Network (CIRN) Provincial Collaborative Network (PCN) Investigators. Effectiveness of BNT162b2 and mRNA-1273 covid-19 vaccines against symptomatic SARS-CoV-2 infection and severe covid-19 outcomes in Ontario, Canada: test negative design study. BMJ2021;374:n1943.

3 Polack FP, Thomas SJ, Kitchin N, etalC4591001 Clinical Trial Group. Safety and Efficacy of the BNT162b2 mRNA Covid-19 Vaccine. N Engl J Med 2020;383:2603-15. doi: 10.1056/NEJMoa2034577 pmid: 33301246

4 Baden LR, El Sahly HM, Essink B, etalCOVE Study Group. Efficacy and Safety of the mRNA-1273 SARS-CoV-2 Vaccine. N Engl/ Med 2021;384:403-16. doi: 10.1056/NEJMoa2035389 pmid: 33378609

5 Tanriover MD, Doğanay HL, Akova M, etalCoronaVac Study Group. Efficacy and safety of an inactivated whole-virion SARS-CoV-2 vaccine (CoronaVac): interim results of a double-blind, randomised, placebo-controlled, phase 3 trial in Turkey. Lancet 2021;398:213-22. doi: 10.1016/S0140-6736(21)01429-X pmid: 34246358

6 Tregoning JS, Flight KE, Higham SL, Wang Z, Pierce BF. Progress of the COVID-19 vaccine effort: viruses, vaccines and variants versus efficacy, effectiveness and escape. Nat Rev Immunol 2021.pmid: 34373623

7 Lopez Bernal J, Andrews N, Gower C, etal. Effectiveness of Covid-19 Vaccines against the B.1.617.2 (Delta) Variant. N Engl J Med2021;385:585-94. doi: 10.1056/NEJMoa2108891 pmid: 34289274

8 Tracking SARS-CoV-2 variants. [cited 6 Aug 2021]. https://www. who.int/emergencies/emergencyhealth-kits/trauma-emergency-surgery-kit-who-tesk-2019/tracking-SARS-CoV-2-variants

9 Verani JR, Baqui AH, Broome CV, etal. Case-control vaccine effectiveness studies: Preparation, design, and enrollment of cases and controls. Vaccine 2017;35:3295-302. doi: 10.1016/j.vaccine.2017.04.037 pmid: 28442231

10 Hungerford D, Vivancos R, Read JM, etal. Mitigating bias in observational vaccine effectiveness studies using simulated comparator populations: Application to rotavirus vaccination in the UK. Vaccine 2018;36:6674-82. doi: 10.1016/j.vaccine.2018.09.051 pmid: 30293764

11 King C, Beard J, Crampin AC, etalVacSurv Consortium. Methodological challenges in measuring vaccine effectiveness using population cohorts in low resource settings. Vaccine 2015;33:4748-55. doi: 10.1016/j.vaccine.2015.07.062 pmid: 26235370

12 Pottegård A, Lund LC, Karlstad Ø, etal. Arterial events, venous thromboembolism, thrombocytopenia, and bleeding after vaccination with Oxford-AstraZeneca ChAdOx1-S in Denmark and Norway: population based cohort study. BMJ 2021;373:n1114 doi: 10.1136/bmj.n1114 pmid: 33952445

13 Perera R, Fletcher J. Thromboembolism and the Oxford-AstraZeneca vaccine. BM/2021;373:n1159. doi: 10.1136/bmj.n1159 pmid: 33952506

14 CDC. COVID-19 Vaccination. Centers for Disease Control and Prevention. 2020 [cited 9 Aug 2021]. https://www.cdc.gov/coronavirus/2019-ncov/vaccines/recommendations/adolescents.html

15 Widianto S. Indonesia recommends Sinovac vaccine for children aged 12-17. Reuters. 2021 Jun 28 [cited 9 Aug 2021]; https://www.reuters.com/world/asia-pacific/indonesia-recommendssinovac-vaccine-children-aged-12-17-2021-06-28/

16 Zou X, Cao B. COVID-19 vaccines for children younger than 12 years: are we ready? Lancet Infect Dis2021;S1473-3099(21)00384-4. https://www.thelancet.com/journals/laninf/article/PIIS14733099(21)00384-4/abstract.pmid: 34197765

17 Han B, Song Y, Li C, et al. Safety, tolerability, and immunogenicity of an inactivated SARS-CoV-2 vaccine (CoronaVac) in healthy children and adolescents: a double-blind, randomised, controlled, phase 1/2 clinical trial. Lancet Infect Dis 2021 published online 28 June [cited 9 Aug 2021]; https://www.sciencedirect.com/science/article/pii/S1473309921003194 